WORKING PAPER · NO. 2020-121

\title{
Uncertainty Spillovers for Markets and Policy
}

Lars Peter Hansen

FEBRUARY 2021 


\title{
Uncertainty Spillovers for Markets and Policy*
}

\author{
Lars Peter Hansen (University of Chicago) ${ }^{\dagger}$
}

February 24, 2021

\begin{abstract}
We live in a world filled with uncertainty. In this essay, I show that featuring this phenomenon more in economic analyses adds to our understanding of how financial markets work and how best to design prudent economic policy. This essay explores methods that allow for a broader conceptualization of uncertainty than is typical in economic investigations. These methods draw on insights from decision theory to engage in uncertainty quantification and sensitivity analysis. Uncertainty quantification in economics differs from most sciences because there is uncertainty both from the perspective of an external observer and from people and enterprises within the model. I illustrate these methods in two example economies in which the understanding of long-term growth is limited. One example looks at uncertainty ramifications for fluctuations in financial markets, and the other considers the prudent design of policy when the quantitative magnitude of climate change and its impact on economic opportunities is unknown.
\end{abstract}

Keywords - uncertainty, valuation, financial markets, policy, climate change, ambiguity, misspecification

*I thank William "Buz" Brock, Doug Diamond, Yiran Fan, Jian Li, Marco Loseto, Lubos Pastor, Diana Petrova, Tom Sargent, Jose Scheinkman, and Grace Tsiang for helpful comments. Financial support for this project was provided by the Alfred P. Sloan Foundation [grant G-2018-11113]. When citing this paper, please use the following: Hansen, L.P., Uncertainty Spillovers for Markets and Policy, https://doi.org/10.1146/annurev-economics-082020054645 .

†email: lhansen@uchicago.edu, ORCID: 0000-0003-0035-8558. 


\section{Introduction}

We must infer what the future situation would be without our interference, and what changes will be wrought by our actions. Fortunately, or unfortunately, none of these processes is infallible, or indeed ever accurate and complete. Knight (1921)

We live in an environment in which uncertainty is pervasive. In the first decade of this century, we experienced a financial crisis. The magnitude of the crisis caught many policymakers, academics, entrepreneurs and businesses by surprise given the previous extended period of a so-called "great moderation." As the COVID-19 pandemic has unfolded at the end of the second decade, we are living through uncertainties such as: i) what exposure we have to the virus when we are in public places, ii) how soon infections will show a permanent decrease, and iii) how soon will a reliable vaccine be available? These and other episodes reveal the limits to our knowledge in ways that have a potentially important impact on the design of policy.

I read Knight's comment from 1921 as a serious challenge to quantitative dynamic modeling in economics. His century-old concern continues to apply today. Economists, as analysts external to their models, face uncertainty "outside the model" when making inferences on limited data. Economic agents, people and businesses, also face uncertainty when making decisions, such as consumption and investment choices, not knowing for sure how the future will unfold. As featured in Hansen (2014), my interest is in models with agents who are cognizant of this "inside the model" uncertainty when making forward-looking decisions. The internal perspective on uncertainty adds an important twist to the external uncertainty quantification that is often addressed in other scientific disciplines.

How then can we capture broad notions of uncertainty in formal ways amenable to quantitative modeling? In this essay, I draw on results from decision theory under uncertainty extended to dynamic settings. Following this literature, I push beyond the risk analyses that are a familiar tool for most economists. Under what I and others call risk, probabilities of future outcomes are known with full confidence but not the outcomes themselves. The broader notion of uncertainty that I embrace includes both ambiguity across alternative possible models and the potential misspecification of each. Under the decision theories that I apply, there can be uncertainty modeling inputs including subjective probabilities as in Bayesian priors over alternative models. Instead of having full confidence in a complete probabilistic specification, a decision maker conducts a sensitivity analysis over families of models or over probabilities assigned to such families. A decision maker explores the consequences of misspecification by broadening substantially the family of models under consideration. Analogous to the familiar aversion to risk, this decision theory introduces an aversion to ambiguity over models or to their potential misspecifications. This aversion effectively dictates how extensive the sensitivity analysis is. The greater the aversion, the less restrictive is

the sensitivity analysis. The approach is made operational by using a decision problem to isolate the most troublesome model or probability over models subject to the restrictions imposed on the sensitivity analysis. 
Rather than delineating axiomatic defenses for the alternative formulations of decision theory under uncertainty, I illustrate their importance in two example economies. Both entail uncertainty about the long-term forces driving the macroeconomy. Section 4 shows how to generate volatility in market valuation by letting investors inside the model be uncertain about future macroeconomic growth prospects. Section 5 studies the social cost of carbon by considering a fictitious social planner assessing the economic consequences of climate change when there is both geoscientific and economic uncertainty.

Before turning to the two applications, in section 2, I discuss more the quantitative modeling with highly stylized models. In section 3 , I show how to use asset pricing theory to quantify the impact of long-term uncertainty in valuation over alternative cash-flow horizons. In section 3 , I construct a martingale component to stochastic discount factor processes used for both market and social valuation. This component dominates valuation adjustments for uncertainty over long horizons and can have important implications over even shorter horizons. The uncertainty adjustments to be characterized in sections 4 and 5 contribute prominently to this martingale component.

\section{Quantitative Storytelling ${ }^{1}$}

"Hominem unius libri timeo (I fear the man of a single book)." St. Thomas Aquinas

Structural models in economic dynamics are meant to be tools for helping us gain a better understanding of a variety of phenomena and to provide guides for designing and evaluating economic policy challenges. The models that interest me in this essay are ones that are quantitative, that allow for random impulses, and hence, are stochastic in nature. Under rational expectations, the economic decision makers within the models recognize the existence of these shocks in the future and know their probabilities. That is, they confront what I (and many predecessors) formally call risk.

I find it attractive to think of each such model as providing a quantitative story. For a lot of questions in economics, I am reminded of the quote of St. Thomas Aquinas mentioned at the outset of this section. Here, I take the liberty to extend this quote to warn any decision maker who is fully committed to one model and its associated story. Thus, for many purposes, I find it valuable to entertain multiple model specifications (reflecting different economic structures or alternative parameter configurations) leading me to quantitative storytelling with "multiple stories." Looking across model specifications or constructions is often done informally and potentially across specific constructions by alternative researchers. ${ }^{2}$ While it may make good sense for this paper to drill

\footnotetext{
${ }^{1}$ The term "quantitative storytelling" has been used elsewhere in a different but partially overlapping way. For instance, see Saltelli and Giampietro (2017). I do not intend to address nor do I fully embrace their critiques of evidence-based policy. But I do share their concern about committing to one model with excessive confidence when drawing on evidence and prior insights to derive optimal policies.

${ }^{2}$ An obvious exception is the extensive literature on Bayesian estimation of stochastic general equilibrium models under rational expectations. This literature confronts unknown parameters with subjective probability inputs, but typically decision makers within these models are presumed to know the unknown parameters in contrast to an econometrician.
} 
down on the implications of a single model, there are many settings in which a decision maker would be wise to look across models produced by different researchers or research groups.

I explore the consequences of doing this more formally and within the actual model construction. In the first of my two substantive examples, I will have investors inside the model entertain parameter uncertainty including the possibility of time-varying parameters governing economic growth. In the second example, a hypothetical policymaker aims to address climate change in the presence of limited knowledge about the impact of $\mathrm{CO}_{2}$ emissions on future economic opportunities.

I use the term ambiguity to capture the uncertainty associated with how much weight should be assigned to the alternative models. One elegant solution supported by an axiomatic defense is the subjective probability approach of de Finetti and Savage. This argues for assigning subjective probabilities or a prior over alternative models, which can be updated by Bayes' theorem based on evidence. But a decision maker who is unsure about how these subjective inputs may wish to explore consequences of this uncertainty. This is the perspective of a robust Bayesian. In some circumstances, the data may be so rich that the posterior probabilities are not very sensitive to the prior inputs, in which case the consequences of ambiguity may be minimal. We might expect this phenomenon to be pervasive in our current "big data" environment with machine learning algorithms helping to assess the data implications. But the data are often not rich along all dimensions of interest. It seems far too severe to limit research questions to ones for which sensitivity over a substantively interesting set of priors is of little consequence. ${ }^{3}$ The examples I choose in this essay are both cases in which I argue prior sensitivity remains an important consideration.

My metaphorical use of the term "story" for a model is made in part because a model is necessarily misspecified. It is a simplification or an abstraction that helps us think about the world or how to design more prudent policies, and it is not intended as a complete accounting of a complex economic environment. Designing omnibus statistical tests for misspecification seems to be a bit "beside-the-point," unless these tests direct researchers to salient improvements in the model specification in substantively interesting ways. Including a rich variety of random shocks to make models harder to reject is a common practice, but it leaves open the question whether these are best conceived of as exogenous impulses or devices to disguise model approximation errors. Excessive shock proliferation can quickly make the model look like a "black box." A harder, but I think important, challenge is systematically to explore the potential consequences of model misspecification. What potential consequences concerned us? To address this question, it is natural to think in terms of a decision problem.

\section{Some asset pricing theory}

The manner in which risk operates upon time preference will differ, among other things, according to the particular periods in the future to which the risk applies. Fisher (1930)

\footnotetext{
${ }^{3}$ Of course, dogmatic priors cannot be dominated by data. In my first example, I entertain such priors as possibilities.
} 
I use an asset pricing perspective to address problems associated with both social and private valuation. As the primary focus of my essay is on uncertainty with long-term consequences, I will exposit a valuation framework with this in mind. This framework will allow me to address two questions:

- When will long-term uncertainty have short-term implications for markets?

- How does discounting compound over long horizons?

It is well-known from the simplest exogenous growth models that both the subjective rate of discount and the intertemporal elasticity of substitution impact how private and social cash flows get discounted optimally or in equilibrium. Uncertainty contributes to discounting as well. A stochastic counterpart replaces the single per-period discount rate because social or private payoffs that are exposed to uncertainty in different ways should be discounted differently. Broadening conceptually the uncertainty that markets and policymakers face alters the stochastic contributions to valuation. Thus, my push beyond the "risk" mentioned in the Irving Fisher quote will be important for the applications that I consider.

\subsection{Prior Literature}

The framework I describe was originally motivated by a quantitative literature in macroeconomics and finance that features contributions of risk and uncertainty to valuation over alternative investment horizons. This entire literature is a direct outgrowth of Rubinstein (1976)'s fundamental paper on valuing flows that grow stochastically over time. The quantitative contributions include, for instance, the work by Parker and Julliard (2003), Campbell and Vuolteenaho (2004), Alvarez and Jermann (2005), Lettau and Wachter (2007), Hansen et al. (2008), Bansal et al. (2009), and van Binsbergen et al. (2012). Much of this quantitative/empirical research worked with log-linear or lognormal approximations. I will not attempt to survey this literature, but instead I will suggest a framework for understanding the valuation implications over alternative investment horizons that is both mathematically convenient and can accommodate nonlinearity. I find this a valuable starting point for thinking about how growth rate uncertainty can impact valuation.

\subsection{Framework}

I represent valuation by taking as given the equilibrium solution to a dynamic, stochastic equilibrium model. I begin with some notation: let $X=\left\{X_{t}: t=0,1, \ldots\right\}$ be a state vector process including, for instance, endogenous capital and exogenous shifts in technology; let $W=\left\{W_{t}: t=1,2, \ldots\right\}$ denote a $k$-dimensional vector of independent and identically distributed shocks to the macroeconomy; and let $\mathfrak{F}_{t}$ represent the information generated by histories of $W$ and the initial state $X_{0}$. For the time-being, I presume that the model has been written in the form of a stochastic steady state, 
and the state vectors to be appropriately scaled to capture this. Thus, for now I take the state vector $X$ to be stationary, although the tools I exposit do not require this restriction. ${ }^{4}$

From the solution of the economic model, I write:

$$
\begin{aligned}
X_{t+1} & =\psi\left(X_{t}, W_{t+1}\right) \\
Y_{t+1}-Y_{t} & =\kappa\left(X_{t}, W_{t+1}\right)
\end{aligned}
$$

where $Y=\left\{Y_{t}: t=0,1, \ldots\right\}$ is a scalar process with stationary increments, and $Y_{0}$ is initialized based on date zero information captured by $\mathfrak{F}_{0}$. The first equation gives the state vector dynamics as a first-order Markov process for the state dynamics. I will use the dynamics in this second equation flexibly to represent alternative economic and financial constructs, including the logarithms of equilibrium consumption, investment or capital, and also the logarithm of stochastically growing cash flows and stochastic discount factors. We take these latter two constructs to be ingredients to valuation.

\subsection{Valuation}

We exploit the recursive structure of the Markov formulation. For valuation, it is important that we work in levels and not logarithms. Let's consider two applications of $\exp (Y)$, a stochastic factor process $S$ and stochastic cash flow process $G$. The product $S G$ has the same mathematical structure. That is, if $\log S$ and $\log G$ have representations given by (1) for a $\kappa_{s}$ and $\kappa_{g}$ respectively, then so does their sum with $\kappa=\kappa_{g}+\kappa_{s}$. Consider the date $t$ price of an asset with payoff $G_{t+\tau}$ :

$$
\begin{aligned}
\pi_{t, t+\tau}(G) & =\mathbb{E}\left[\left(\frac{S_{t+\tau}}{S_{t}}\right) G_{t+\tau} \mid \mathfrak{F}_{t}\right] \\
& =\mathbb{E}\left[\left(\frac{S_{t+\tau} G_{t+\tau}}{S_{t} G_{t}}\right) \mid \mathfrak{F}_{t}\right] G_{t} .
\end{aligned}
$$

In these formulas, $\frac{S_{t+\tau}}{S_{t}}$ is the stochastic discount factor over a horizon $\tau$ starting from date $t$. The valuation of equity sums over such prices for $\tau=1,2, \ldots$.

With this computation in mind, we are led to study conditional expectations of the product $G S$ as well as the $S$ and $G$ components over alternative horizons in constructing proportional uncertainty compensations as in Hansen (2012). I express these in logarithms to obtain:

$$
\rho_{t, t+\tau}(G)=\left(\frac{1}{\tau}\right)\left(\log \mathbb{E}\left[\left(\frac{G_{t+\tau}}{G_{t}}\right) \mid \mathfrak{F}_{t}\right]-\log \mathbb{E}\left[\left(\frac{S_{t+\tau} G_{t+\tau}}{S_{t} G_{t}}\right) \mid \mathfrak{F}_{t}\right]+\log \mathbb{E}\left[\left(\frac{S_{t+\tau}}{S_{t}}\right) \mid \mathfrak{F}_{t}\right]\right)
$$

The measure, $\rho_{t, t+\tau}(G)$. is the logarithm of the ratio of two expected returns, one with pay-off $G_{t+\tau}$, and the other one with risk-less payoff normalized to be one. To see this, observe that the first term on the right is the expected payoff contribution, and the second one is the corresponding

\footnotetext{
${ }^{4}$ For the application to climate change that I develop in section 5, I necessarily feature the transition dynamics, but I continue to exploit the Markov specification.
} 
cost of a claim to this pay-off. As the second one comes with a negative sign, the two terms taken together give the logarithm of the expected return over horizon $\tau$. The negative of the third term is the logarithm of the risk-free return over horizon $\tau$, and thus provides a risk-less comparison with the same horizon.

This uncertainty compensation measure, $\rho_{t, t+\tau}(G)$, depends on both the horizon $\tau$ and the conditioning information $\mathfrak{F}_{t}$ available at time $t$. In particular, the dependence on $\tau$ reflects the impact of compounding. The compensation depends on the uncertainty in both the pay-off process $G$ and the stochastic discount factor $S$. The stochastic discount factor contribution reflects the price impact, and this will be of particular interest in this essay.

\subsection{A revealing factorization}

To set the stage for future discussion, I describe a factorization result that was originally formalized by Hansen and Scheinkman (2009) and motivated by previous research of Alvarez and Jermann $(2005) .{ }^{5}$ I write the process $\exp (Y)$ as the product of three terms:

$$
\exp \left(Y_{t}-Y_{0}\right)=\exp (\eta t)\left(\frac{M_{t}}{M_{0}}\right)\left[\frac{e\left(X_{0}\right)}{e\left(X_{t}\right)}\right]
$$

where $M$ is a positive martingale for which $\log M$ has stationary increments with a stochastic evolution given by (1) with an appropriate choice of $\kappa$. In comparison, $\log e(X)$ inherits the stationarity of $X$. Notice that $M$ and $e$ are both only defined up to scale. The real number $\eta$ gives a long-term growth rate or decay rate depending on application. For instance, $\eta$ is negative when $\exp (Y)=S$.

As stated, this representation may not be unique. For applications and for establishing the uniqueness of the factorization, it is most advantageous to use $M$ to induce a change in probability measure. Since $M$ is a positive martingale:

$$
\mathbb{E}\left(\frac{M_{t+1}}{M_{t}} \mid \mathfrak{F}_{t}\right)=1
$$

In light of this outcome, we use an approach that is familiar in the pricing of derivative claims by building a different probability measure with conditional expectation:

$$
\widetilde{\mathbb{E}}\left(B_{t+1} \mid \mathfrak{F}_{t}\right)=\mathbb{E}\left[\left(\frac{M_{t+1}}{M_{t}}\right) B_{t+1} \mid \mathfrak{F}_{t}\right]
$$

where $B_{t+1}$ is a bounded random variable in the date $t+1$ conditioning information of decisionmakers in the economic model. It may be verified that this change in probability measure preserves the same Markov structure as the original probability measure. There is, at most, one such factorization for which this change of measure implies a Markov process that is stochastically stable

\footnotetext{
${ }^{5}$ While Hansen and Scheinkman (2009) derive their result in a continuous-time environment, there is a straightforward extension to discrete-time settings. Qin and Linetsky (2020) relax the Markovian assumption in their extension of Hansen and Scheinkman (2009).
} 
by which I mean that the conditional expectations converge in the limit to well-defined unconditional expectations. This stability property is what allows us to use the factorization to provide meaningful long-term characterizations of pricing.

I remark briefly on the long-term pricing implications of factorization (3) for limiting uncertainty compensation (2).

$$
\lim _{\tau \rightarrow \infty} \rho_{t, t+\tau}(G)
$$

Let $\eta_{g}$ be the growth rate of the cash flow, $\eta_{s}$ the decay rate of the stochastic discount factor and $\eta_{g s}$ the decay rate of the product $G S$. Then:

$$
\lim _{\tau \rightarrow \infty} \rho_{t, t+\tau}(G)=\eta_{g}+\eta_{s}-\eta_{g s}
$$

In the special case in which the stochastic discount factor process does not have a martingale component, this limiting compensation is zero. More generally, the probability associated with the martingale component of $S$ is the long-term forward measure as it absorbs the long-term compensations for exposure to uncertainty. This is also the measure that the Ross (2015) approach would recover in this setting. ${ }^{6}$

In this essay, I will be primarily interested in the martingale component to a stochastic discount factor process. There are three ways to justify such a component:

- permanent shocks to the macroeconomy,

- recursive utility preferences that feature forward-looking components,

- subjective beliefs, ambiguity-averse preferences, or concerns about model misspecification.

I will consider two example economies in which all three components are interconnected and reinforcing. But before doing so, let me elaborate on each and describe why they are related. Speculation about potential macroeconomic growth provides an interesting channel for uncertainty. Macroeconomists debate about the prospects of secular stagnation, and economic historians make different conjectures about the future prospects for technological advances. What are the long-term consequences of climate change on economic opportunities? Will potential future pandemics alter permanently how economies provide goods and services in the future? As emphasized by Bansal and Yaron (2004), the recursive utility model of Kreps and Porteus (1978), Epstein and Zin (1989), and others contribute a forward-looking contribution to even a one-period stochastic discount factor $\frac{S_{t+1}}{S_{t}}$. While these are risk-based models, the same implication is often true in models of ambiguity aversion and model misspecification fears. As a decision-maker grapples with uncertainty about the model specification, long-term uncertainty can emerge as having the biggest adverse impact on their discounted utility. Thus, once again concerns about the unknown nature of the longterm uncertainty can impact even the valuation of short-term assets such as one-period returns. The pricing outcomes of broadly-conceived uncertainty aversion are conveniently represented by

\footnotetext{
${ }^{6}$ See Borovička et al. (2016) for more discussion.
} 
an altered probability specification capturing the uncertainty that impacts decision-making in the most adverse way.

While factorization (3) has a variety of applications, I use it here and feature the interaction between all three rationales for the martingale component to valuation and illustrate its quantitative importance.

\section{Uncertainty and financial markets}

... a practical theory of the future, ... being based on so flimsy a foundation, it is subject to sudden and violent changes. Keynes (1937)

In this section, I describe an example economy in which investors confront uncertainty about the future of macroeconomic growth. I base this example on a paper by Hansen and Sargent (2020a). ${ }^{7}$ This example captures in a stylized way both the worrisome secular stagnation feared by some macroeconomists and the uncertain prospects for technological advances debated by economic historians. The model has a long-run risk component, like that featured by Bansal and Yaron (2004), entering into the evolution of capital. We include production to provide substantively interesting interpretations of shocks. While there is an extensive literature that builds on or applies the Bansal and Yaron risk-based formulation of uncertain growth, the risk aversion that is imposed on investors is typically very large. In the example economy, I show how a broader notion of uncertainty aversion, including model ambiguity and misspecification concerns, provides a different perspective of how uncertain growth impacts asset valuation. While the long-run risk literature typically introduces stochastic volatility, specified exogenously, I abstract from that in the model that I describe. I adopt this simplification to feature what fluctuations in asset valuation can be induced by investor struggles with pinpointing the precise nature of the uncertainty.

As I will show, prices of uncertainty fluctuate because investors especially fear high persistence of macroeconomic growth in bad states, and fear low persistence in good ones. Why this asymmetry? When an economy is growing, the salient fear preoccupying investors is that high growth will not persist. But when the economy is stagnant, the salient fear is that sluggishness will persist. We show how these concerns manifest themselves in terms of both model ambiguity and concerns about model misspecification. Formally, they take the form of an endogenous nonlinearity in the stochastic discount factor that investors use to evaluate the prospective payout streams that underlie asset evaluations. This nonlinearity compounds itself over time in ways that provide a novel explanation for how asset market fluctuations reflect uncertainty.

\footnotetext{
${ }^{7}$ See, also, Hansen and Sargent (2019) for a short perspective on this research.
} 


\subsection{Setup}

To fix ideas, suppose that capital, $K_{t}$, evolves according to the continuous-time stochastic differential equation as:

$$
d K_{t}=K_{t}\left(\alpha_{k}+\beta_{k} Z_{t}+\frac{I_{t}}{K_{t}}\right) d t+K_{t} \sigma_{k} \cdot d W_{t}
$$

where output is proportional to the capital stock:

$$
C_{t}+K_{t} \phi\left(\frac{I_{t}}{K_{t}}\right)=\mathrm{a} K_{t}
$$

$C_{t}$ is consumption, $I_{t}$ is investment, $\phi$ is a convex function introduced to capture adjustment costs, and $d W_{t}$ is a bivariate increment to a Brownian motion. I interpret capital broadly to include potentially human and organization capital.

For simplicity, I assume that investors have a unitary elasticity of intertemporal substitution in preferences, which implies that, for an optimal resource allocation, consumption and capital should be proportional. The process $Z$ captures exogenous shifts in technology and is modeled as a scalar autoregression:

$$
d Z_{t}=\alpha_{z}-\beta_{z} Z_{t}+\sigma_{z} \cdot d W_{t}
$$

While the $Z$ process captures a form of "long-run risk," I have purposefully abstracted from including a stochastic volatility component, even though such a component is common in macro-finance models. While there is evidence for such volatility in the macroeconomy, my aim is to show how concerns about uncertainty can induce fluctuations in the market price of uncertainty endogenously without imposing it through an external source.

There are two parameters that will be of particular interest to us. The first is $\beta_{k}>0$, which determines how responsive the capital stock is to changes in the persistent technology process. Larger values of $\beta_{k}$ imply more exposure to technological uncertainty. The second is $\beta_{z}>0$, which determines the persistence of the process $Z$. Larger values of $\beta_{z}$ imply more mean reversion, and more pull towards the long-run mean. The counterpart to the discrete-time autoregression coefficient is $\exp \left(-\beta_{z}\right)$, which is closer to zero when $\beta_{z}$ is large.

I construct the rational expectations competitive solution by following Lucas and Prescott (1971) approach for which the equilibrium resource allocation is the outcome of a fictitious planner's problem for a Brock and Mirman (1972)-style stochastic growth model. I then compute the three additional solutions:

- planner's problem with a concern for the misspecification of a baseline model used in the rational expectations solution,

- planner's problem with a concern for ambiguity across models as indexed by the parameter $\operatorname{pair}\left(\beta_{k}, \beta_{z}\right)$,

- planner's problem with a concern for both model misspecification and ambiguity across models. 
The first illustrates the impact of robust preferences as in Hansen and Sargent (2001); the second, the impact of ambiguity averse preferences as in Chen and Epstein (2002); and the third, the impact of combining the two as in Hansen and Sargent (2020a). ${ }^{8}$

I treat the case with a unitary elasticity of substitution for pedagogical simplicity. Capital is the sole source of wealth in this economy, and with this preference restriction, consumption is proportional to capital. Similarly, there is a constant investment-capital ratio. While changing how investors confront uncertainty alters the equilibrium stochastic discount factor, the equilibrium allocations for consumption and capital stay the same. I still solve a planner's problem because the minimizing probabilities from the planner's problem, expressed as a function of the state variables and Brownian shocks, contribute an uncertainty adjustment to the stochastic discount factor used in represent asset prices in a competitive equilibrium. ${ }^{9}$ Relative to the rational expectations model, each of the three solutions induces a different martingale contribution. The martingale contributions capture the uncertainty-adjusted probabilities deduced from the planner's problem. Since I posed the model in continuous time, the value functions and worst-case probabilities solve HamiltonJacobi-Bellman equations as in Hansen and Sargent (2020a).

Had I not imposed a unitary elasticity of substitution, or had I introduced multiple capital stocks with differential exposure to uncertainty, the quantity allocation would no longer be invariant across the three alternative preference specifications. Instead, changing the preferences as described in the previous paragraph would have altered not only the stochastic discount factor, but also the equilibrium allocation of consumption and capital. ${ }^{10}$ Such generalizations provide some additional insights in how uncertainty aversion alters precautionary motives for savings as reflected in the evolution of capital. In particular, the ratio of consumption to aggregate capital would be statedependent. Of course, such outcomes are of considerable interest, but here I have chosen to focus on the implications for the equilibrium stochastic discount factor.

\subsection{Modeling details}

Given the equivalence of the capital and consumption dynamics, I construct the baseline parameter values from the estimates of $\left(\widehat{\alpha}_{k}, \widehat{\beta}_{k}, \widehat{\sigma}_{k}, \widehat{\alpha}_{z}, \widehat{\beta}_{z}, \widehat{\sigma}_{z}\right)$ as in Hansen and Sargent (2020a). They set $\hat{\alpha}_{z}=0$ which means that the $Z$ process has mean zero in the implied stationary distribution, and the sum of $\hat{\alpha}_{k}$ plus the investment-capital ratio gives the long-term growth rate in logarithms for consumption and capital. They also set $\widehat{\beta}_{k}=1$. In the rational expectations equilibrium, the investor has full confidence in the baseline parameter values.

To explore model misspecification, the investor considers alternative probability specifications each of which can be represented as a Brownian motion with drift. We use $U$ to represent the

\footnotetext{
${ }^{8}$ The robust preferences used by Hansen and Sargent (2001) and the generalization in Hansen and Sargent (2020a) can be viewed as special cases of the dynamic variational preferences of Maccheroni et al. (2006).

${ }^{9}$ While it is common to compute the competitive equilibrium allocation by solving a social planner's problem, I use an extended version of that same approach to compute the uncertainty adjustment for the equilibrium asset prices. See Hansen and Sargent (2020a) for more elaboration on this point.

${ }^{10}$ See Eberly and Wang (2012) for a tractable extension of this model with two capital stocks and risk-based recursive utility.
} 
stochastic process of drifts. Then:

$$
d \widehat{W}_{t}=U_{t} d t+d W_{t}^{U}
$$

where $d W_{t}^{U}$ is a Brownian motion under the change of probability measure. Thus, locally I am changing the distribution of the underlying shocks from being multivariate standard normal shocks to shocks that have a mean that is not zero and instead can be time and state-dependent. I use flexible specifications of the drift process $U$ to capture alternative forms of misspecification.

The martingale associated with this drift distortion has particularly simple dynamic evolution under the baseline $\hat{\cdot}$ dynamics:

$$
d M_{t}^{U}=M_{t}^{U} U_{t} \cdot d \widehat{W}_{t}
$$

where the (at least local) martingale property follows since the conditional mean or drift is zero. As $M^{U}$ is a likelihood ratio, the evolution of the logarithm is also of particular interest:

$$
\begin{aligned}
d \log M_{t}^{U} & =-\frac{\left|U_{t}\right|^{2}}{2} d t+U_{t} \cdot d \widehat{W}_{t} \\
& =\frac{\left|U_{t}\right|^{2}}{2} d t+U_{t} \cdot d W_{t}^{U}
\end{aligned}
$$

Thus, the local mean of expected log-likelihood ratio under the $M^{U}$ probability distribution is one half the norm squared of the drift distortion $U$. This is the local contribution to relative entropy, which is a well-known discrepancy and mathematically convenient measure for assessing differences in probability measures. Relative entropy is also often referred to as Kullback-Lieibler divergence. This local contribution of relative entropy exploits the underlying Brownian formulation with locally normal shocks. When the squared norm of the drift is large, the implied probability measure is easy to distinguish from the baseline probability.

Motivated by results from the robust control theory, Hansen and Sargent (2001) include penalty that is a scaled version of:

$$
\frac{1}{2} \mathbb{E}\left[\int_{0}^{\infty} \exp (-\delta t)\left(\frac{M_{t}^{U}}{M_{0}^{U}}\right)\left|U_{t}\right|^{2} d t \mid \mathfrak{F}_{0}\right]
$$

in the objective function of the fictitious planner. ${ }^{11}$ This objective includes the same subjective discount rate, $\delta$, as is used in discounting expected utility of the planner. I impose the restriction so that the preferences are dynamically consistent. A larger penalty scaling limits more the potential consequences of model misspecification to the decision-maker. Including this term in the planner's objective leads to a particularly simple choice of $U_{t}^{*}$ for this example economy:

$$
U_{t}^{*} \propto-\left(\frac{\widehat{\beta}_{k}}{\widehat{\beta}_{z}+\delta}\right) \widehat{\sigma}_{z}-\widehat{\sigma}_{k} .
$$

The right-hand side of this expression gives the negative of how the increment in the continuation

\footnotetext{
${ }^{11}$ For some related robust control references, see Jacobson (1973), James (1992), and Petersen et al. (2000).
} 
value (expressed in logarithms) responds to the Brownian increment. Notice that both slope parameters contribute to this drift distortion. More exposure to the exogenous growth rate uncertainty, captured by a larger value $\widehat{\beta}_{k}$, magnifies the contribution of $\hat{\sigma}_{z}$. More persistence in this process captured by a smaller value of $\widehat{\beta}_{z}$ has the same qualitative impact. The proportionality factor depends on the reciprocal of the scaling factor for the relative entropy penalty. In other words, a large penalty results in a small implied drift distortion. Notice that while the drift distortion can be state-dependent, in the solution from the planner's problem, this distortion is constant. The decision-maker repeatedly worries about permanent shifts in drift of the Brownian increment. Since $-U_{t}^{*}$ is constant, this gives a mechanism for enhancing uncertainty prices but not for causing them to vary. While this invariance is driven in part by the functional forms I have assumed, more generally I expect the implied state dependence in the uncertainty prices to be small.

I now consider ambiguity aversion relative to what Hansen and Sargent (2020a) call a structured set of models. This will also illustrate a version of the Chen and Epstein (2002) formulation of ambiguity aversion. In this computation, to capture parameter ambiguity, I introduce a special case of the drift distortion:

$$
S_{t}=\eta_{0}+\eta_{1} Z_{t}
$$

where $\eta_{0}$ and $\eta_{1}$ are two-dimensional vectors. Now rewrite state vector dynamics as:

$$
\begin{aligned}
d K_{t} & =K_{t}\left(\widehat{\alpha}_{k}+\widehat{\beta}_{k} Z_{t}+\frac{I_{t}}{K_{t}}\right) d t+K_{t} \widehat{\sigma}_{k} \cdot d \widehat{W}_{t} \\
& =K_{t}\left(\alpha_{k}+\beta_{k} Z_{t}+\frac{I_{t}}{K_{t}}\right) d t+K_{t} \widehat{\sigma}_{k} \cdot d W_{t}^{S} \\
d Z_{t} & =\widehat{\alpha}_{z}-\widehat{\beta}_{z} Z_{t} d t+\widehat{\sigma}_{z} \cdot d \widehat{W}_{t} \\
d Z_{t} & =\alpha_{z}-\beta_{z} Z_{t} d t+\widehat{\sigma}_{z} \cdot d W_{t}^{S} .
\end{aligned}
$$

where:

$$
\begin{array}{ll}
\alpha_{k}=\hat{\alpha}_{k}+\hat{\sigma}_{k} \cdot \eta_{0} & \beta_{k}=\widehat{\beta}_{k}+\widehat{\sigma}_{k} \cdot \eta_{1} \\
\alpha_{z}=\hat{\alpha}_{z}+\hat{\sigma}_{z} \cdot \eta_{0} & \beta_{z}=\hat{\beta}_{z}+\widehat{\sigma}_{z} \cdot \eta_{1}
\end{array}
$$

By varying $\eta_{0}$ and $\eta_{1}$, I obtain alternative parameters of the mean dynamics of the state variables.

Given the role that $\widehat{\beta}_{k}$ and $\widehat{\beta}_{z}$ in the previously constructed $U^{*}$, I will feature ambiguity about these two parameters. Thus, I hold fixed $\left(\alpha_{k}, \sigma_{k}, \alpha_{z}, \sigma_{z}\right)=\left(\widehat{\alpha}_{k}, \hat{\sigma}_{k}, 0, \hat{\sigma}_{z}\right)$ and limit the parameter uncertainty to $\left(\beta_{k}, \beta_{z}\right)$. This leads me to focus on $\eta_{1}$ and set $\eta_{0}=0 .{ }^{12}$ As Hansen and Sargent (2020b) argue, I cannot embed misspecification concerns of the type described previously within this framework, because Chen and Epstein (2002) impose an instant-by-instant constraint. They restrict Brownian motion drifts in this manner to implement a version of Gilboa and Schmeidler (1989) ambiguity-averse preferences extended to be dynamically consistent. To implement this approach, we continue to use relative entropy but now in a much more limited way.

Consider for the moment a single alternative model given by a choice of $\eta_{1}$. I could compute

\footnotetext{
${ }^{12}$ Hansen and Sargent (2020a) also present results when $\eta_{0}$ is different from zero.
} 
the implied discounted relative entropy $\epsilon$, which will be dependent on the realized state $z$. My aim, however, is to build a constraint on structured models. Thus, I start with a function $\epsilon$ and find values of $\eta_{1}$ 's with the same relative entropy. This defines the boundary of the set of structured models and is characterized by a quadratic equation in the two-dimensional vector $\eta_{1}$ or equivalently for $\left(\beta_{k}, \beta_{z}\right)$. I also include the interior points, each of which is a drift distortion associated with a value of $\eta_{1}$, in this ambiguity set. Given this constraint, the planner will justifiably focus on the boundary when minimizing over $S$. As Hansen and Sargent (2020a) make clear this constraint on $S$ is much tighter than imposing a date zero constraint that implied relative entropy be less than or equal to $\epsilon\left(Z_{0}\right)$.

The fictitious planner's problem is actually a two-player max-min dynamic game that can expressed and solved using recursive methods. The maximization is over investment and minimization, conditioned on the investment choice, is over the structured drift distortion. For the recursive minimization, I start with a functional equation for relative entropy for each $\eta_{1}$ on the boundary. ${ }^{13}$ I convert this equation into a constraint on $\eta_{1}$ for each possible value of $z$ by taking the relative entropy $\epsilon$ as a function of $z$ as input. For my application, this minimization problem is quadratic given the state $z .^{14}$

Without getting more distracted in the details, I depict the resulting constraints on the parameters in Figure 1.

\footnotetext{
${ }^{13}$ This equation is a special case of the so-called Feynman-Kac equation.

${ }^{14}$ See Hansen and Sargent (2020a) for more details.
} 


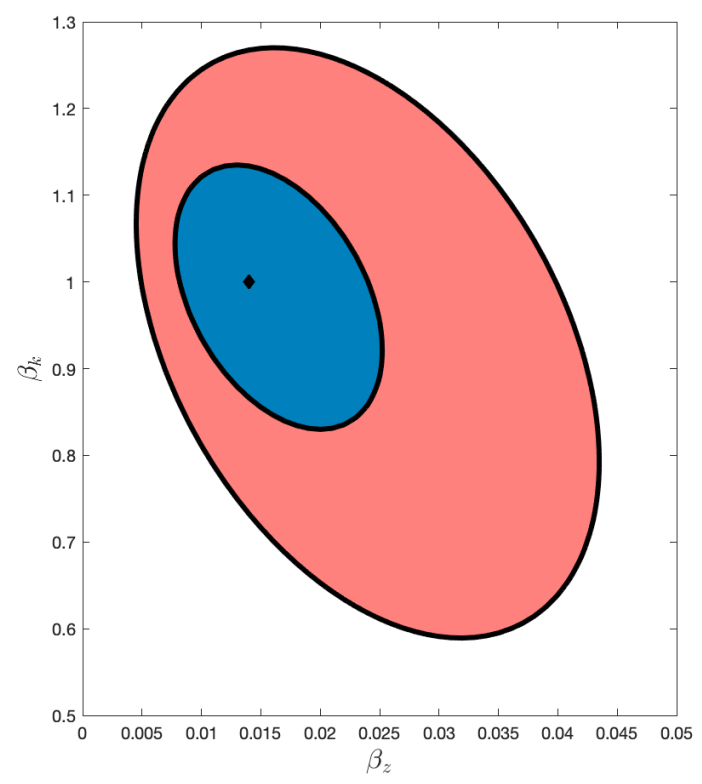

Figure 1: Ambiguity sets: parameter values constrained by relative entropy where $\beta_{k}$ quantifies exposure to the macro growth-rate process and $\beta_{z}$ quantifies the persistence of that process. The single point is the baseline specification $\left(\widehat{\beta}_{k}, \widehat{\beta}_{z}\right)$ and the two regions are implied by a relatively tight and a relative loose relative entropy constraint. This figure is essentially the same as Figure 2 from Hansen and Sargent (2020a). Its use is authorized under a Creative Commons CC-BY-NC-ND license.

The decision-maker is more ambiguity-averse when the ambiguity set is larger. To be consistent with Chen and Epstein (2002), I allow for the minimizing choice of $\left(\beta_{k}, \beta_{z}\right)$ to depend on the alternative realized values $z$ of $Z_{t}$. This is indeed an outcome of our model solution. Following Hansen and Sargent (2020a), I motivate this extra flexibility by allowing a priori the parameters to be time-varying but constrained to reside in the ambiguity parameter set. Alternatively, I could allow for a restricted set of nonlinear specifications of the local means for the state-vector evolution.

Investors in this economy start with a simple linear first-order autoregressive model of macroeconomic growth rate dynamics. Their concerns about ambiguity induce them to explore other statistically similar specifications including ones that they especially fear. In Figure 2, the linear relation with the negative slope captures their baseline model with so-called mean reversion for the growth rate process $Z$. The mean reversion is evident because there is a pull from the more extreme growth states towards the centre of the growth rate distribution. The vertical axis is the local pull towards the centre of the distribution of macroeconomic growth (net of its long-run average growth rate.) In the absence of random shocks, there is a pull towards zero. In the left panel, I also include a kinked red line that is the ambiguity-averse response when investors confront a set of statistically similar specifications. Formally, they explore the ambiguity set of parameters for different macroeconomic growth states. The flatter slope (a smaller value of $\beta_{z}$ ) to the left of 
zero reflects the concerns of investors in bad economic times that the macroeconomy may be stuck with more growth sluggishness than in the original model. The steeper slope to the right of zero reflects opposite forces. Here, good macroeconomic growth outcomes are feared to be shorter-lived than in the original model specification. Finally, in the left panel we plot the implied drift when the only investor aversion is that the baseline model is misspecified, which just shifts the baseline drift proportionally downward. There is a corresponding movement in the minimizing choice of $\beta_{k}$, which governs how exposed the economy to the uncertain growth process.
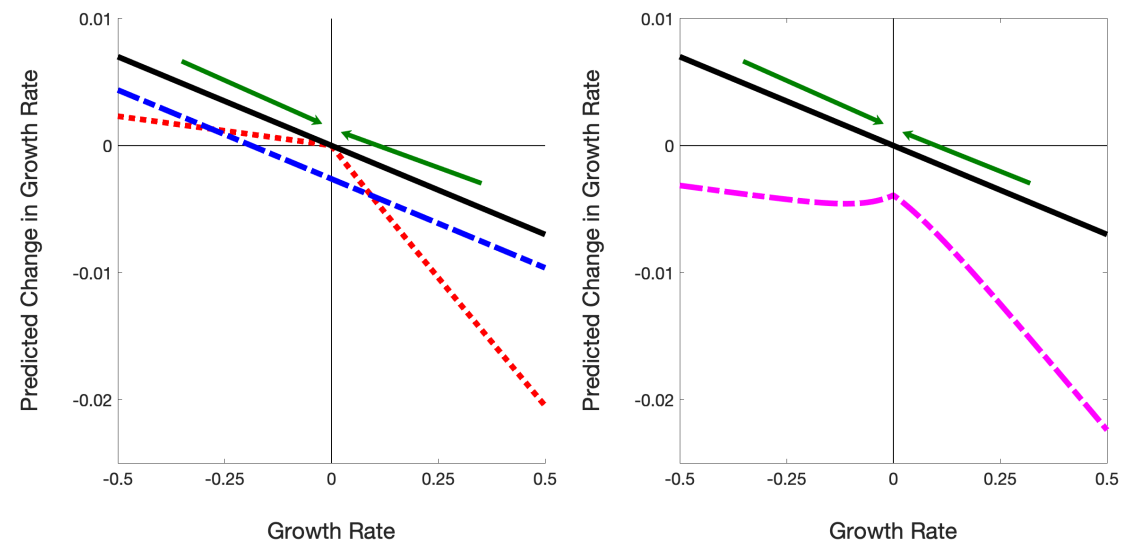

Figure 2: Local dynamics for macroeconomic growth. Black solid line represents the baseline linear model. In the left panel, the red dotted curve incorporates aversion to model ambiguity and the blue dashed line incorporates aversion to model misspecification. In the right panel, the magenta dashed curve incorporates aversions to both model ambiguity and model misspecification. This is adapted from Figure 3 of Hansen and Sargent (2020a). Its use is authorized under a Creative Commons CC-BY-NC-ND license.

Neither the red dotted curve nor the blue dashed line are intended to represent the "beliefs" of the planner or the investors inside our example economy. The computation of this altered drift is part of deducing the averse response to ambiguity over the state dynamics. The imputed drift distortion $S^{*}$ from the planner's problem that implements the competitive equilibrium is a nonlinear function of the growth state $Z$. Interestingly, it is a continuous-time version of a threshold autoregression model. The martingale associated with this $S^{*}$, with a drift given by:

$$
d M_{t}^{S^{*}}=S_{t}^{*} \cdot d \widehat{W}_{t}
$$

contributes prominently to the martingale component of the stochastic discount factor.

In the right panel of Figure 2, I look at a specification featured by Hansen and Sargent (2020a) that allows each model in the ambiguity set to be misspecified. Here, the structured specifications of the drift distortion $S$ are in the same ambiguity set described previously, but I also include an 
relative entropy penalty that is proportional to:

$$
\frac{1}{2} \mathbb{E}\left[\int_{0}^{\infty} \exp (-\delta t)\left(\frac{M_{t}^{U}}{M_{0}^{U}}\right)\left|U_{t}-S_{t}\right|^{2} d t \mid \mathfrak{F}_{0}\right] .
$$

The unstructured misspecification is captured by the drift distortion $U$, which is penalized relative to the set of structured models. The right panel of Figure 2 shows the resulting minimizing unstructured drift distortion given by the magenta dashed curve. The altered drift inherits a similar shape to that ambiguity-adjusted drift, but it is shifted downward. This downwards shift is no longer proportional, as it was when there was only a single specification considered by investors.

Empirical evidence suggests that uncertainty compensations are larger in bad times for the macroeconomy relative to good times. The local uncertainty compensation induced by an aversion to ambiguity or model misspecification is governed by $U^{*}$. To explore the pricing effects, we consider a term structure of uncertainty prices captured by the formula:

$$
\mathbb{E}\left(M_{t}^{U^{*}} U_{t}^{*} \mid Z_{0}\right)
$$

I think of these as building blocks for valuation adjustments due to uncertainty. Notice that $-\sigma_{k} \cdot U^{*}$, as depicted in the right panel of Figure 2, is large when the magnitude of the growth state is large. This is true independent of whether growth is relatively high or relatively low. An important asymmetry emerges, however, once we look at the horizon dependence reflected in formula (5). The steeper sloping conditional mean for $Z$ when $z$ is positive means that under the $M^{U^{*}}$ probability there is a relatively fast escape from these states vis-a-vis the baseline probability specification. Conversely, there is a slower escape for $z$ negative. This asymmetry is reflected in Figure 3 which reports the term structure of uncertainty prices given by (5). These uncertainty prices depend on the current growth state. We consider three values of this state, one is median $(\mathrm{z}=0)$ and the others are the .1 and .9 deciles of the $Z$ stationary distribution under the base probability measure. While both the upper and lower deciles start substantially higher than the median uncertainty prices, the .9 decile prices show substantial decay because of the likely escape from the high growth state. The two panels give the uncertainty prices for the two underlying shocks. Notice that the patterns are very similar. In summary, the term structure reveals an intriguing asymmetry in the uncertainty prices depending on macroeconomic growth. 

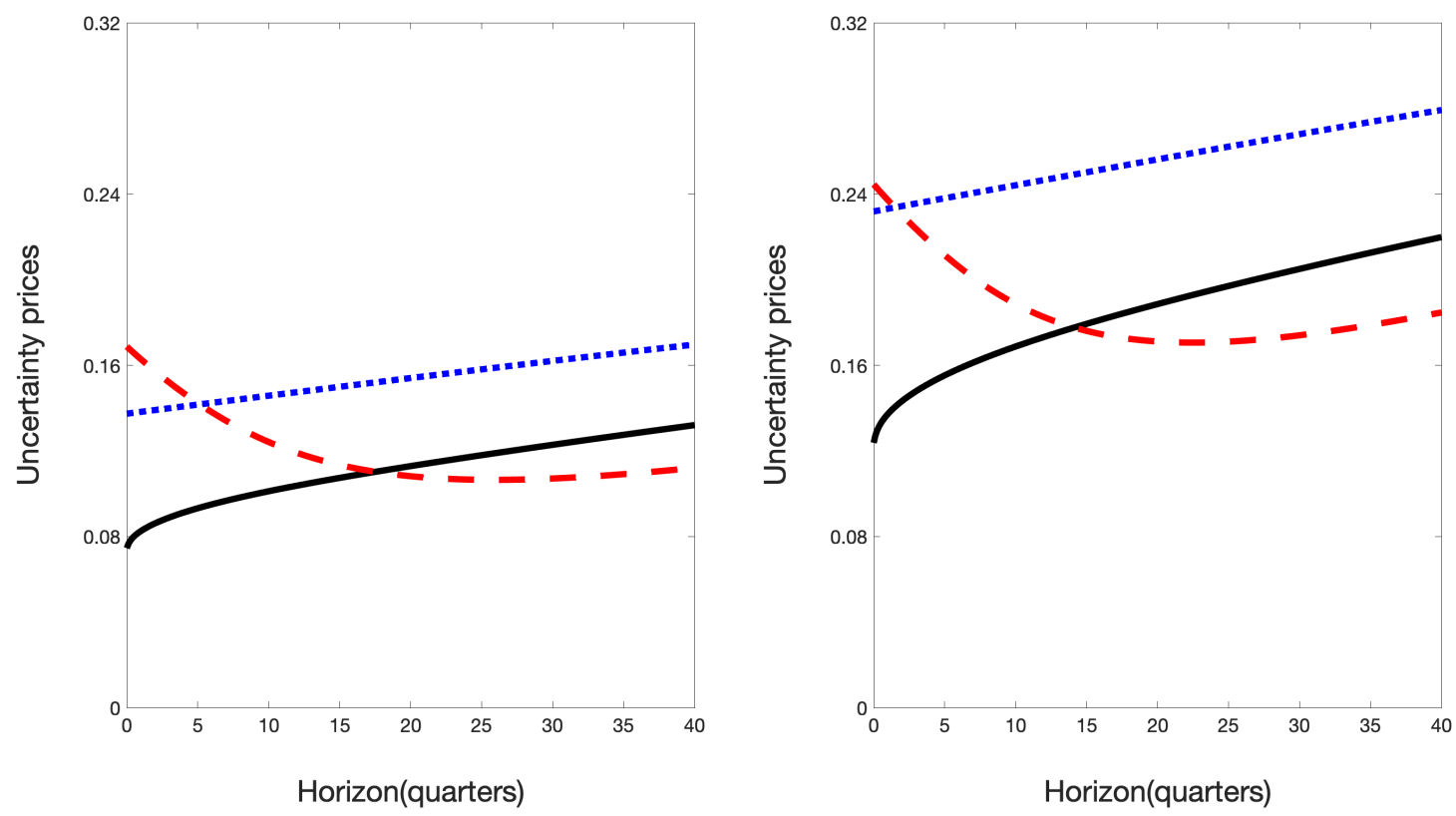

Figure 3: Term structure of uncertainty prices. The black curve sets the initial growth state at the median of the stationary distribution; the red dashed curve sets the initial state at the .9 decile; the blue dotted line sets the initial state at the .1 decile. The two panels give the term structure of prices for exposures to the two underlying shocks. This figure is extracted from Figure 6 in Hansen and Sargent (2020a). It use is authorized under a Creative Commons CC-BY-NC-ND license.

\subsection{Related literature}

In this example economy, I abstracted from learning by including alternative models with parameters whose prospective variations cannot be inferred from historical data. These time-varying parameter models differ from the decision-maker's baseline model, a fixed parameter model whose parameters can be well estimated from historical data. Other research imposes parameter invariance and instead induces fluctuations using a robust Bayesian perspective. For instance, in Hansen and Sargent (2010) and Hansen (2007) a representative investor's robust model averaging to drive fluctuations in uncertainty prices. The investor carries along difficult-to-distinguish models of macro growth. The investors make robust adjustments to Bayesian posteriors updated with historical data. This leads the investors to act as if good news is temporary and bad news is persistent, an outcome that is qualitatively similar to what we have found here. The decision-makers in their analyses confront dynamic consistency by playing a game against future versions of themselves, although I speculate that similar qualitative insights could be obtained using the continuous-time recursive formulation in Hansen and Miao (2018). Collin-Dufresne et al. (2016) and Andrei et al. (2019) showed that a comparable outcome emerges with a risk-based, recursive utility formulation with Bayesian learning in which investors have full confidence in the subjective probabilities and are not concerned about model misspecification. While it is valuable to see this rational learning 
counterpart, the risk-based approaches miss part of what I consider to be an important challenge facing investors as they speculate about the future.

\section{$5 \quad$ Uncertainty and policy}

Even if true scientists should recognize the limits of studying human behavior, as long as the public has expectations, there will be people who pretend or believe that they can do more to meet popular demand than what is really in their power. Hayek (1974)

There is often a tendency to overstate the knowledge base when providing public rationales for

policies aimed at addressing social and economic problems. Premising decisions on false knowledge can lead to bad outcomes. Some might argue that unless we know for sure (or at least with great confidence), we should adopt a wait-and-see approach. But this conclusion does not necessarily follow from the basic tenets of decision theory under uncertainty. Since there can be a substantial cost to the delay, decision theory can justify acting now based on the possibility of bad outcomes in the future, should we fail to act. The aversion to uncertainty is meant to resolve a trade-off in assessing decisions between focusing on best guesses and considering possible adverse outcomes even though they might be unlikely. My hope is that tools such as the ones described in this essay can allow policymakers to confront uncertainty in sanguine discussions of policy.

In this section, I will explore the impact of uncertainty in assessing the economic uncertainty of climate change by drawing on the analysis in Barnett et al. (2020). In this research, they assess a stylized "integrated assessment model," incorporating both geoscientific and economic uncertainty. There exist many such integrated assessment models, but my particular focus will be on the consequences of uncertainty for prudent policy-making. Following Barnett et al., I show that geoscientific and economic uncertainties are in effect multiplicative in quantifying the social cost of carbon (SCC) emissions. I accomplish this by using the tools from asset pricing to study social valuation in contrast to private valuation. A central part of their, and hence, my analysis is the construction of probability measures that adjust the uncertainties pertinent to valuation. Specifically, I construct a martingale component to the stochastic discount factor based on a cautious approach to uncertainty.

\subsection{Social Cost of Carbon (SCC)}

The SCC is commonly referred to in policy discussions, but its meaning and implication for measurement differ across applications. In this discussion, I will use a well-posed version as an analytical tool to assess the impact of uncertainty. I alter the technology in the previous section to include an exploration sector that leads to increases in the stock of fossil fuel reserves. In addition, I will modify preferences to include a demand for emissions. Emissions will alter the climate, which in turn will impact economic opportunities and social well-being in the future. I take the emissions impact on the climate and economic opportunities to be an externality not captured by market 
prices. I model the SCC as a stochastic process of the implied shadow prices for emissions pertinent for a fictitious social planner engaged in socially efficient resource allocation. The SCC in my computations depends on the underlying state variables in the economy, and thus, varies over time. The wedge between the market prices and the SCC is the process of so-called Pigouvian taxes on emissions that would "correct" the externality.

Pigouvian tax policy could be dismissed as "pie-in-the-sky," and the use of a single social planner as "remarkably naive," since it ignores a variety of practical challenges in policymaking. I am sympathetic to such criticisms, and I will not address the many political and economic challenges of climate change policy. Instead, I use the SCC as a barometer for gauging the quantitative importance of uncertainty.

I bring in tools from asset pricing by viewing the SCC as a process of asset prices with a corresponding social cash flow. For simplicity, I will use temperature as the measure of climate change. The social cash flows of interest have two interconnected contributions:

- geoscience: how do changes in $\mathrm{CO}_{2}$ emissions alter temperature in the future?

- economics: how do changes in temperature alter economic opportunities in the future?

Both of these contributions can be conceived of as nonlinear, local impulse responses, the first being the impulse response of emissions for future temperature changes, and the second being the impulse response of temperature to future changes in economic opportunities. In effect, I form a convolution of these two response functions to get the social cash flow. The SCC agglomerates the social cash flows using stochastic discounting including a martingale adjustment for uncertainty.

The social marginal rate of substitution between emissions and consumption is commonly referred to as the social cost of carbon (SCC). Thus it is a shadow price of the resource allocation problem for a hypothetical planner. It could be implemented via a Pigouvian tax that would correct the private shadow price for the externality, although we use this way to assess the impact of uncertainty, when conceived broadly. Following previous literature, we start by representing this social cost in terms of partial derivatives of the value function of the social planner. We then apply an asset pricing perspective to interpret components to this social cost.

\subsection{Prior Literature}

As many previous researchers have investigated, the human impact on the climate is a potentially important source of uncertainty that could play out over long horizons. See for instance, Weitzman (2012), Jensen and Traeger (2014), Cai et al. (2015), Nordhaus (2017), Hambel et al. (2018) and especially Cai et al. (2017). Our use of an asset pricing perspective to interpret the SCC follows in part discussions in Golosov et al. (2014) and Cai et al. (2017), who have an ambitious exploration of the risk consequences for the social cost of carbon. My aim is to show how to extend the analyses to include forms of uncertainty other than risk. While I use a particular model of the climatesystem for the sake of illustration, this same perspective also allows researchers to understand 
better the components to the social cost applicable in more general settings. Millner et al. (2013) and Lemoine and Traeger (2016), previously used the smooth ambiguity formulation of decision making motivated by climate science. In contrast to the analysis that follows, they did not motivate their calibration of ambiguity formally by robustness and sensitivity considerations.

\subsection{Setup}

I use an AK model of the type I specified in the previous section (see equation (4)), except that I modify the output constraint to be:

$$
C_{t}+I_{t}+J_{t}=\alpha K_{t}
$$

where $J_{t}$ is investment in the discovery of new fossil fuel reserves. The stock of reserves evolves according to:

$$
d R_{t}=-E_{t} d t+\psi_{0}\left(R_{t}\right)^{1-\psi_{1}}\left(J_{t}\right)^{\psi_{1}}+R_{t} \sigma_{R} \cdot d W_{t}
$$

where $R_{t}$ is the date $t$ stock of reserves, and $E_{t}$ is the date $t$ flow of emissions of fossil fuels drawn from the reserve stock. The parameters satisfy the restrictions $\psi_{0}>0$ and $0<\psi_{1} \leqslant 1$. The well-known Hotelling (1931) model of resource extraction with a fixed stock of reserves becomes a special case of our reserves by setting $\psi_{0}=0$, and $\sigma_{R}=0$ and restricting $R_{t} \geqslant 0$ for all $t \geqslant 0 .{ }^{15}$

Capturing the externality induced by climate change requires the integration of a climate change component to the model. It is intractable to integrate a complex and high-dimensional climate model with an even stylized economic model of the type that I use here. To get off the ground, I adopt some seemingly stark approximations. Climate scientists have performed a variety of model comparisons by using exogenously specified representative concentration pathways and by using emission pulses of different sizes. Examples are found in Eby et al. (2009), Joos et al. (2013), and MacDougall et al. (2017). The simplified dynamics are often specified in terms of two subsystems: i) the dynamic response of atmospheric carbon concentration to emissions and ii) the dynamic response of temperature to atmospheric carbon concentration. The response of temperature to emissions is in effect a convolution of these two responses. While it is common for integrated assessment models to formally specify these two subsystems, there is a substantial literature that studies direct simplifications of the convolution by showing that the temperature change over an interval of time is roughly proportional to the cumulative carbon emissions over the same time period. Matthews et al. (2009), among other contributions, proposed this as a useful approximation. Many subsequent papers have assessed this approximation and have used it for model comparisons and policy discussion. For instance, Ricke and Caldeira (2014) have argued that, in fact, this proportionality emerges over a relatively short timescale. Dietz and Venmans (2019) and Barnett et al. (2020) in independent contributions, have used this approximation within

\footnotetext{
${ }^{15}$ Our introduction of a technology for investing the exploration of new reserves follows from previous contributions by Casassus et al. (2018) and Bornstein et al. (2019). Instead of investing in new discoveries, Nordhaus (2008)-style (DICE) models introduce investing as a way to make emissions more productive in the technology for output.
} 
an explicit economic optimization framework. The latter contribution proposes ways to confront uncertainty, broadly-conceived, within such a framework. The Barnett et al. paper uses the crossmodel uncertainty in the proportionality relationship reported as input into their analysis. See the left panel of Figure 4 for the cross-model histogram reported in the MacDougall et al. paper for the proportionality coefficient $\beta$. Taking this histogram as a direct characterization of uncertainty means that all of the inputs into the construction of the histogram are given equal weighting. While this might be an interesting starting point, we shall examine sensitivity to this choice.

From the economic side, I specified what is called a "damage function" meant to summarize the economic consequences of climate change. While there are good reasons to consider dynamic responses to climate change through alternative forms of adaptation, like much of the literature, including Dietz and Venmans (2019) and Barnett et al. (2020), I posit a static relationship between temperature and damages, denoted by $N_{t}$. I follow Barnett et al. by letting $N_{t}$ capture a proportional reduction in consumption due to climate change. Formally, they assume that:

$$
\log N_{t}=\Gamma\left(\beta \int_{0}^{t} E_{u} d u\right)+\zeta_{n}\left(Z_{t}\right)
$$

where:

$$
\Gamma(y)=\left\{\begin{array}{cc}
-\gamma_{1} y-\frac{\gamma}{2} y^{2} & y \leqslant 2 \\
-\gamma_{1} y-\frac{\gamma}{2} y^{2}-\frac{\gamma_{2}^{+}}{2}(y-2)^{2} & y>2
\end{array}\right.
$$

The parameters $\gamma_{2}$ and $\gamma_{2}^{+}$give nonlinear damage adjustments. Specifically, the $\gamma_{2}^{+}>0$ gives a smooth alternative to a carbon budget at two degrees celsius. Notice that the function $\Gamma$ is zero at zero, decreasing and concave. ${ }^{16}$ We include an exogenous shifter in this specification of damages via $\zeta_{n}\left(Z_{t}\right)$.

There is substantial divergence in the environmental economics literature on magnitude of damages. To illustrate this divergence, the right-hand side Figure 4 depicts $\exp [\Gamma(y)]$ for two different possible $\gamma_{2}^{+}$, one is zero and the other is positive. The construction of the $\Gamma$ with $\gamma_{2}^{+}=0$ is motivated by the evidence reported in Nordhaus and Moffatt (2017), and the second more curved specification of $\Gamma$ with $\gamma_{2}^{+}>0$ is loosely motivated by Weitzman (2012)'s arguments for much more severe damages beyond two degrees. Given this specification of damages, we see that $\gamma_{2}^{+}$is multiplied by either $\beta$ or $\beta^{2}$, implying that the uncertainty across the economic and climate inputs is multiplicative. In the illustrative calculation, I impose a baseline subjective probability of equal weight on the two values of $\gamma_{2}^{+}$, and explore the consequences of making arguably small changes in these probabilities.

\footnotetext{
${ }^{16}$ As in Barnett et al. (2020), I posit $\Gamma$ as a function of the climate change component to temperature fluctuations by omitting other exogenous components of temperature change not induced by economic activity.
} 

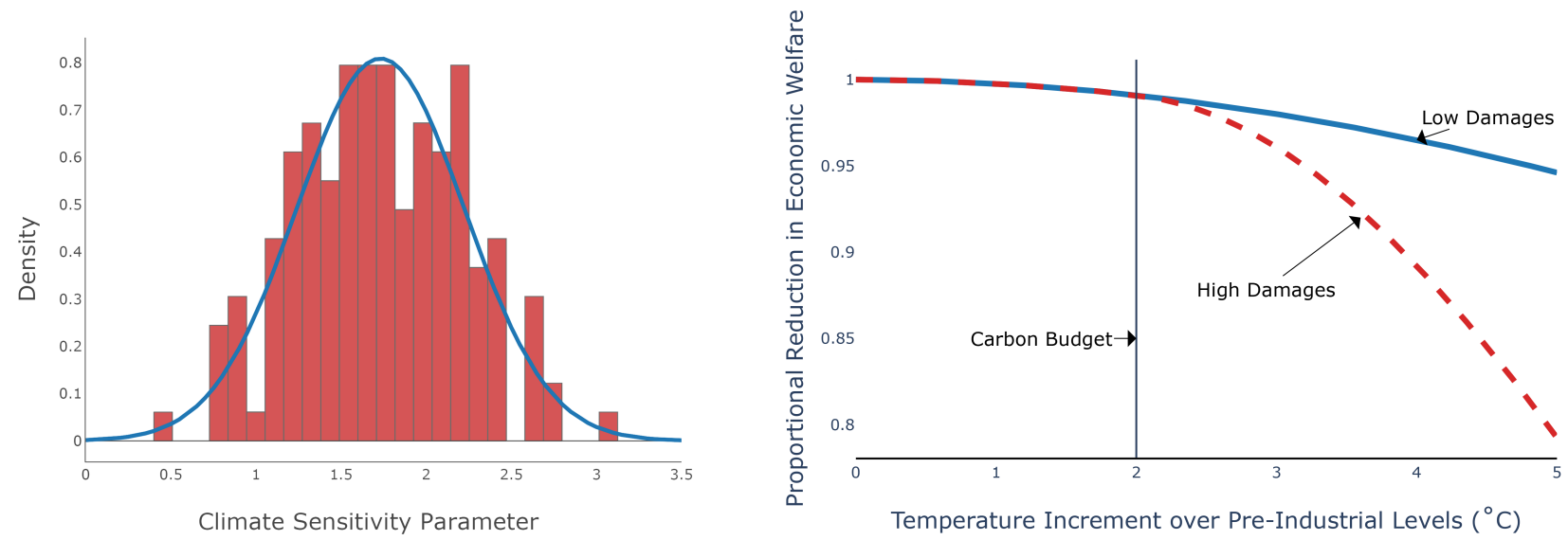

Figure 4: Uncertainty inputs. Left panel: Histograms and density for the climate sensitivity parameter across models. The Figure is based on evidence reported in Figure 3A by MacDougall et al. (2017) (C) American Meteorological Society, used with permission) and constructed with data provided by the authors. I use the blue curve as a baseline specification of probabilities across models instead of red histogram to simplify computations. Right panel: Two specifications of the damage functions specified as a proportional reduction in the consumption to changes due to climate change measured as the climate sensitivity parameter times cumulative emissions. This figure is essentially the same as Figure 3 in Barnett et al. (2020). It use is authorized under a Creative Commons CC-BY-NC-ND license.

The fictitious planner in our analysis confronts ambiguity in the distribution of the climate sensitivity parameter $\beta$ and the damage parameter $\gamma_{2}^{+}$. The planner uses baseline subjective probabilities for both. We use the Hansen and Miao (2018) framework for a recursive implementation of the smooth ambiguity model in continuous time. The discrete-time version of smooth ambiguity was originally axiomatized by Klibanoff et al. (2005). Hansen and Sargent (2007) provide a robust Bayesian interpretation of an important special case of the discrete-time smooth ambiguity model, whereby the impact of changes in the baseline posterior probabilities are explored recursively subject to relative entropy penalty. ${ }^{17}$ The Hansen and Miao (2018) formulation preserves this robustness interpretation. Like Hansen and Sargent (2007), Hansen and Miao also show how to extend this decision theory representation to include concerns about model misspecification. In the climate economic computations that I report, I feature only the model ambiguity component. This is in contrast to the example of uncertainty impacts for financial markets that I described in the previous section. The robust Bayesian interpretation provides an altered probability as part of the planner solution for resource allocation, giving rise to a martingale component to a stochastic discount factor pertinent for social valuation. ${ }^{18}$

\footnotetext{
${ }^{17}$ While the example I report considers two potential damage functions, Li et al. (2016) consider a parameterized family of log-linear damage functions with a baseline exponential distribution over the unknown slope parameter. They follow Hansen and Sargent (2007) by making a robustness adjustment to this baseline distribution.

${ }^{18}$ Initial applications of smooth ambiguity models to the economics of climate change can be found in Millner et al. (2013) and Lemoine and Traeger (2016), but without reference to robustness considerations for the subjective
} 


\subsection{Implications of Ambiguity for the SCC}

While stochastic discounting has been extensively applied in market valuation, it is also pertinent in social valuation. It justifiably redirects discussions about what should be the discount rate for social valuation. Just as in market valuation, there is a stochastic discount factor that adjusts valuation for exposures to uncertainty in ways that can depend on both the horizon and current Markov state. Again, the martingale component is of particular interest. As in market valuation under uncertainty, there is a measure that adjusts for aversions to ambiguity and model misspecification associated with this martingale component.

The SCC is an asset price associated with a particular social cash flow that reflects the adverse impact of climate change on economic and social outcomes. ${ }^{19}$ This social cash flow is the impulse response function of emissions today on damages in the future where damages incorporate marginal utility adjustments from the different time periods. We then apply an asset pricing perspective to interpret components to this social cost. This social cash flow depends on the interacting uncertainty about economic damages and climate change. Following Barnett et al. (2020), I include the ambiguity adjusted probabilities from the robust planner's problem. This change of probability measure contributes a martingale component to the valuation. ${ }^{20}$

In what follows, I report some calculations from Barnett et al. (2020). They abstract from learning about unknown parameters and assume that the baseline probabilities for the climate sensitivity parameter, $\beta$, and that those for the damage parameter, $\gamma_{2}^{+}$, are invariant over time. The damage uncertainty pertains to damages induced by temperature for which we do not have historical data. This severely limits the possibility of Bayesian learning. For the parameter $\beta$, learning is also particularly challenging. The cross-model uncertainty that showed in Figure 4 seems difficult to be noticeably reduced in the near future. While abstracting from learning is a reasonable starting point, it may well be that if we start to experience even more extreme temperatures and more severe damages, then evidence in the future could become much more informative and helpful in resolving this uncertainty. Even though the baseline probabilities are time invariant for the calculations I report, the probability adjustments are time-varying. This follows from the recursive formulation of the decision problem. Specifically, the consequences of changing baseline probabilities become more pronounced as damages increase with warmer temperatures, as we see in Figure 5. This is evident from adjusted densities reported at year 50 versus year 100. By year 100, the high damage specification, $\gamma_{2}+>0$, is assigned probability .59 in contrast to .5 for the baseline probability and for the year 50 probability.

probabilities.

${ }^{19}$ Such a discussion follows in part from Golosov et al. (2014). Cai et al. (2017) have a more ambitious exploration of the risk consequences for the SCC. Barnett et al. (2020) also embrace an asset pricing interpretation, but in particular, they show how to extend the analysis of the SCC to include aversions to ambiguity and model misspecification.

${ }^{20}$ While I use mathematical methods familiar from asset pricing theory, the implied shadow prices are computed using a socially efficient allocation and not necessarily an observed allocation determined by suboptimal policies and competitive markets. 

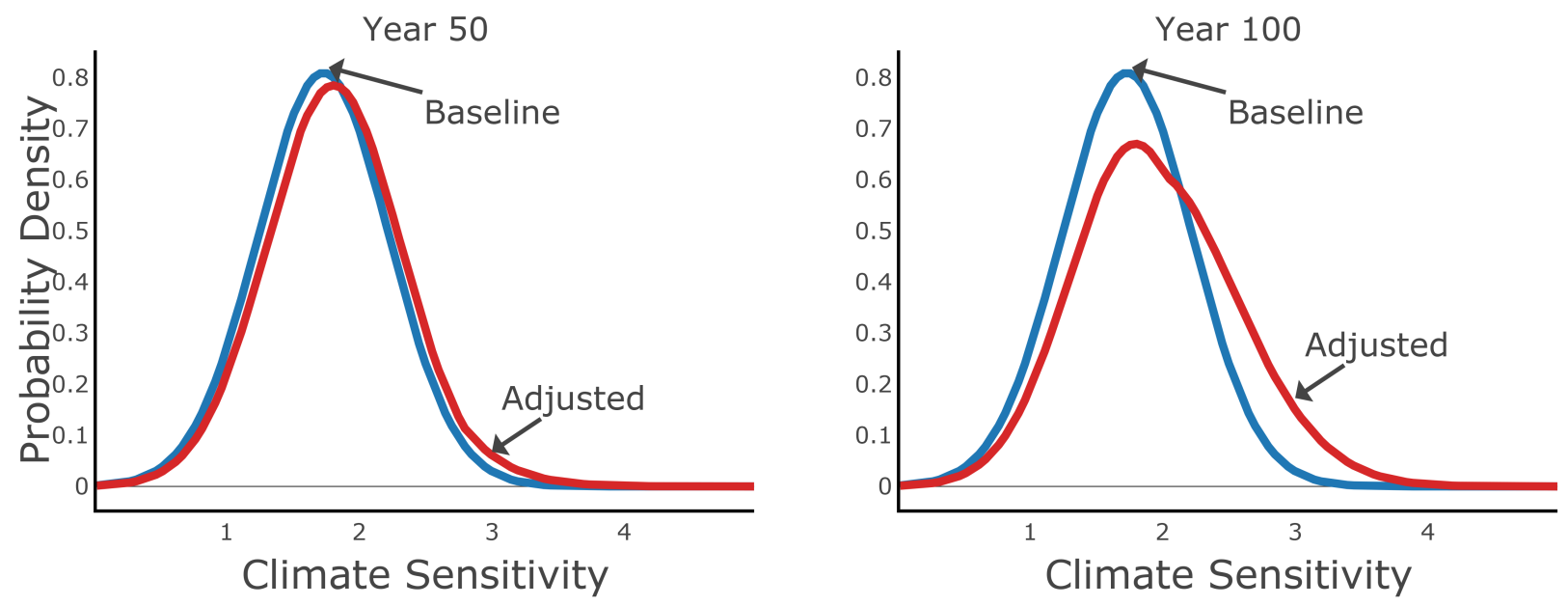

Figure 5: The initial weighting is the same for both the low- and high-damage specifications. The ambiguity-adjusted probabilities remain very close to this at year 50, but are tilted towards the high damage specification (probability .59) at year 100. This figure is essentially the same as Figure 3 in Barnett et al. (2020). Its use is authorized under a Creative Commons CC-BY-NC license.

While the probability adjustments displayed in Figure 5 may seem modest, they have a quantitatively important impact on the SCC as depicted in Figure 6. This latter figure reports the SCC's using stochastically discounted social cash flows with expectations computed with two distinct probabilities. One uses the uncertainty-adjusted probabilities, and the other uses the baseline probabilities. The former is the correct calculation and includes a martingale component needed to adjust for ambiguity about how to subjectively weight the alternative climate model outcomes, and how to weight the two alternative specifications of damages. The increases in the SCC for both trajectories, while reminiscent of Hotelling (1931), are induced by the potential impact of damages to economic opportunities in the future in conjunction with the continued economic growth and not to a finite stock of fossil fuel resources. 


\section{Social Cost of Carbon Decomposition}

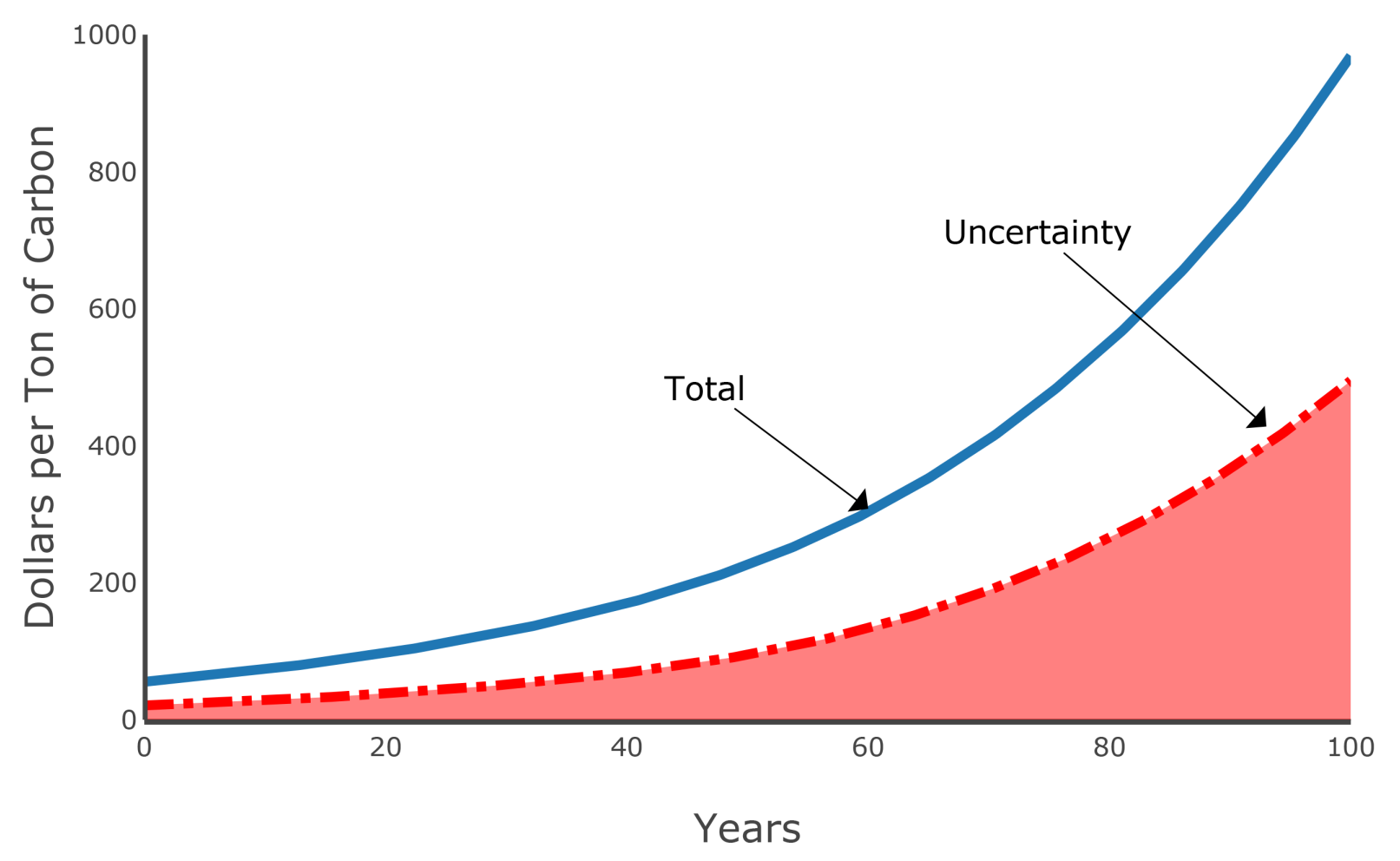

Figure 6: SCC computed under two probability measures. The blue solid curve gives the SCC for the ambiguity averse planner. The vertical difference between the blue solid curve and the red dashed curve gives the counterpart computation with the same social cash flow but using the benchmark probabilities without the martingale adjustment. The red dashed curve is what we call the uncertainty contribution. This figure is adapted from Figure 6 in Barnett et al. (2020). It use is authorized under a Creative Commons CC-BY-NC license.

The model used in this section is purposely stylized to show how uncertainty can impact the SCC. An enhanced version of the model, while computationally more demanding, could address the uncertainty consequences for potential future policy and private sector responses to climate change. These could include the transition from fossil fuel to renewable energy or green technologies; the potential for increasing the sink capacity through biodiversity conservation; and the endogenous economic responses to climate change including adaptation. These extensions will open additional channels through uncertainty that will impact social and economic outcomes, making the tools discussed in this essay all the more relevant. 


\section{Conclusion}

For a variety of economic phenomena, researchers, policymakers, and economic agents are hardpressed to come up with a single model or lens to use for an enhanced understanding or for the design of a prudent policy. The tools that I described preserve tractability while allowing for decision making when there is ambiguity over models and the potential of each to be misspecified. I used the term "quantitative storytelling" metaphorically to capture the process of using a model with empirically motivated inputs to make quantitative predictions without carrying the burden of being correctly specified. This essay addresses the need to balance off the implications of multiple stories in decision making.

I presented two examples of quantitative storytelling with multiple stories to show how this uncertainty impacts the analysis and the conclusions. Empirical asset pricing researchers will have little difficulty finding asset pricing anomalies that the first example fails to address. But my point in developing this example is to exposit a novel mechanism without the external clutter needed to address a broader set of empirical evidence from financial markets. While the aim of the second example is to quantify the SCC, there have been challenges made by others as to the value of integrated assessment models used for this purpose. For instance, Pindyck (2013) and Morgan et al. (2017) argue that SCC calculations from integrated assessment models are of limited value for setting policy because of the modeling simplifications and the limited understanding of climate change and its potential for damaging the economy. The methods I describe are meant to open the door to incorporating a better characterization of the uncertainties and knowledge limits we encounter. With the simplified modeling inputs that I used in the illustration, some important policy-relevant questions are off the table. Nevertheless, the methods that I illustrate open the door to a variety of important extensions that will enlarge the scope of the analysis while continuing to acknowledge the uncertainties.

I offer up the methods described here to reveal the sensitivity of quantitative conclusions to model ambiguity and potential misspecification. The penalty parameters needed as inputs tie directly to (decision-makers') uncertainty aversions. A researcher using these methods need not choose a single configuration of these parameters. Here, we can start with a broad array of model specifications and subjective probabilities. The decision problem, in conjunction with credible subjective inputs and empirical evidence, reveals where the subjective inputs have the biggest impact on the decision-making of economic agents or policymakers. The quantitative implications depend on penalization parameters, but the approach ensures that dependence will be of lowdimension. Inspired by Good (1952), I apply robust Bayesian methods modified to be applicable to the study of economic dynamics. A methodological achievement of the research I describe is providing a framework for showing how parsimonious penalized probabilities reveal sensible choices on how to restrain the search over subjective inputs.

The two examples that I explore align the uncertainty perspectives of private agents in the model with those of a fictitious planner. In the first example, this alignment is used to simplify the computation of the competitive equilibrium prices. In presenting the second application, I suggest, 
but do not formally derive, a competitive equilibrium counterpart subject to Pigouvian taxes deduced from a planner's problem. More generally, however, the planner and private agents may have different perspectives on what is uncertain. It is common in macroeconomics to study the design and implementation of policies by formulating Ramsey-type problems as dynamic games played between a government and private agents. Hansen and Sargent (2012) formulate and compare such problems with three different types of ambiguity. In two of three formulations, the heterogeneity between a government and private agents in terms of the ambiguities that they confront are reflected in the resulting equilibria. Karantounias (2013) uses one of these formulations to study how a government that is fully confident in its probability model of its expenditures conducts fiscal policy when facing a skeptical public. In a dynamic, hidden-action contracting problem, Miao and Rivera (2016) posit a principal who has doubts about probabilities when engaging a better informed and more confident agent. Importantly, these examples open the door to the study of the impact that heterogeneous perspectives on ambiguity among agent types in an economy has on contract and policy design. 


\section{References}

Alvarez, Fernando and Urban J. Jermann. 2005. Using Asset Prices to Measure the Persistence of the Marginal Utility of Wealth. Econometrica 73 (6):1977-2016.

Andrei, Daniel, Michael Hasler, and Alexandre Jeanneret. 2019. Asset Pricing with Persistence Risk. The Review of Economic Studies 32 (7):2809-2849.

Bansal, Ravi and Amir Yaron. 2004. Risks for the Long Run: A Potential Resolution of Asset Pricing Puzzles. Journal of Finance 59 (4):1481-1509.

Bansal, Ravi, Robert Dittmar, and Dana Kiku. 2009. Cointegration and consumption risks in asset returns. Review of Financial Studies 22 (3):1343-1375.

Barnett, Michael, William A. Brock, and Lars Peter Hansen. 2020. Pricing Uncertainty Induced by Climate Change. Review of Financial Studies 33 (3):1024-1066.

Bornstein, Gideon, Per Krusell, and Sergio Rebelo. 2019. Lags, Costs, and Shocks: An Equilibrium Model of the Oil Industry. NBER Working Paper 23423, National Bureau of Economic Research, Inc.

Borovička, Jaroslav, Lars Peter Hansen, and José A. Scheinkman. 2016. Misspecified Recovery. Journal of Finance 71 (6):2493-2544.

Brock, W and L Mirman. 1972. Optimal Economic Growth and Uncertainty: The Discounted Case. Journal of Economic Theory 4 (3):479-513.

Cai, Yongyang, Kenneth L Judd, Timothy M Lenton, Thomas S Lontzek, and Daiju Narita. 2015. Environmental Tipping Points Significantly Affect the Cost-Benefit Assessment of Climate Policies. Proceedings of the National Academy of Sciences 112 (15):4606-4611.

Cai, Yongyang, Kenneth L. Judd, and Thomas S. Lontzek. 2017. The Social Cost of Carbon with Climate Risk. Tech. rep., Hoover Institution, Stanford, CA.

Campbell, John Y. and Tuomo Vuolteenaho. 2004. Bad Beta, Good Beta. American Economic Review 94 (5):1249-1275.

Casassus, Jaime, Pierre Collin-Dufresne, and Bryan R. Routledge. 2018. Equilibrium Commodity Prices With Irreversible Investment and Non-Linear Technologies. Journal of Banking and Finance 95:128-147.

Chen, Zengjing and Larry Epstein. 2002. Ambiguity, Risk, and Asset Returns in Continuous Time. Econometrica 70 (4):1403-1443.

Collin-Dufresne, Pierre, Michael Johannes, and Lars A. Lochstoer. 2016. Parameter Learning in General Equilibrium: The Asset Pricing Implications. American Economic Review 106 (3):664698. 
Dietz, Simon and Frank Venmans. 2019. Cumulative carbon emissions and economic policy: In search of general principles. Journal of Environmental Economics and Management 96:108-129.

Eberly, Janice C and Neng Wang. 2012. Reallocating and Pricing Illiquid Capital: Two Productive Trees. Tech. rep., Columbia University and Northwestern University.

Eby, M., K. Zickfeld, A. Montenegro, D. Archer, K. J. Meissner, and A. J. Weaver. 2009. Lifetime of anthropogenic climate change: Millennial time scales of potential CO2 and surface temperature perturbations. Journal of Climate 22 (10):2501-2511.

Epstein, Larry G. and Stanley E. Zin. 1989. Substitution, Risk Aversion and the Temporal Behavior of Consumption and Asset Returns: A Theoretical Framework. Econometrica 57 (4):937-969.

Fisher, Irving. 1930. Theory of Interest. New York: The Macmillan Company.

Gilboa, Itzhak and David Schmeidler. 1989. Maxmin Expected Utility with Non-Unique Prior. Journal of Mathematical Economics 18 (2):141-153.

Golosov, Mikhail, John Hassler, Per Krusell, and Aleh Tsyvinski. 2014. Optimal Taxes on Fossil Fuel in General Equilibrium. Econometrica 82 (1):41-88.

Good, Irving J. 1952. Rational Decisions. Journal of the Royal Statistical Society. Series B (Methodological) 14 (1).

Hambel, Christoph, Holger Kraft, and Eduardo Schwartz. 2018. Optimal Carbon Abatement in a Stochastic Equilibrium Model with Climate Change. Working Paper 21044, National Bureau of Economic Research.

Hansen, Lars Peter. 2007. Beliefs, Doubts and Learning: Valuing Macroeconomic Risk. American Economic Review 97 (2):1-30.

. 2012. Modeling the Long Run: Valuation in Dynamic Stochastic Economies. Econometrica $80(3): 911-967$.

- 2014. Nobel Lecture: Uncertainty Outside and Inside Economic Models. Journal of Political Economy 122 (5):945-987.

Hansen, Lars Peter and Jianjun Miao. 2018. Aversion to Ambiguity and Model Misspecification in Dynamic Stochastic Environments. Proceedings of the National Academy of Sciences 115 (37):9163-9168.

Hansen, Lars Peter and Thomas J. Sargent. 2001. Robust Control and Model Uncertainty. The American Economic Review 91 (2):60-66.

- 2007. Recursive Robust Estimation and Control without Commitment. Journal of Economic Theory 136 (1):1-27. 
. 2010. Fragile Beliefs and the Price of Uncertainty. Quantitative Economics 1 (1):129-162.

—. 2012. Three Types of Ambiguity. Journal of Monetary Economics 59 (5):422-445.

_. 2019. Acknowledging and Pricing Macreconomic Uncertainties. VOXEU.

- 2020a. Macroeconomic Uncertainty Prices when Beliefs are Tenuous. Journal of Econometrics publisehd online, November.

- 2020b. Structured Ambaiguity and Model Misspecification. Journal of Economic Theory published online, December.

Hansen, Lars Peter and Jose A. Scheinkman. 2009. Long-Term Risk: An Operator Approach. Econometrica 77 (1).

Hansen, Lars Peter, John C. Heaton, and Nan Li. 2008. Consumption Strikes Back? Measuring Long-Run Risk. Journal of Political Economy 116 (2):260-302.

Hayek, F.A. 1989. The Pretense of Knowledge. The American Economic Review 79 (6):3-7.

Hotelling, Harold. 1931. The Economics of Exhaustible Resources. Journal of Political Economy 39 (2):137-175.

Jacobson, David H. 1973. Optimal Stochastic Linear Systems with Exponential Performance Criteria and Their Relation to Deterministic Differential Games. IEEE Transactions for Automatic Control AC-18:1124-1131.

James, Matthew R. 1992. Asymptotic Analysis of Nonlinear Stochastic Risk-Sensitive Control and Differential Games. Mathematics of Control, Signals and Systems 5 (4):401-417.

Jensen, Svenn and Christian Traeger. 2014. Optimal Climate Change Mitigation under LongTerm Growth Uncertainty: Stochastic Integrated Assessment and Analytic Findings. European Economic Review 69 (C):104-125.

Joos, F., R. Roth, J. S. Fuglestvedt, G. P. Peters, I. G. Enting, W. Von Bloh, V. Brovkin, E. J. Burke, M. Eby, N. R. Edwards, T. Friedrich, T. L. Frölicher, P. R. Halloran, P. B. Holden, C. Jones, T. Kleinen, F. T. Mackenzie, K. Matsumoto, M. Meinshausen, G. K. Plattner, A. Reisinger, J. Segschneider, G. Shaffer, M. Steinacher, K. Strassmann, K. Tanaka, A. Timmermann, and A. J. Weaver. 2013. Carbon Dioxide and Climate Impulse Response Functions for the Computation of Greenhouse Gas Metrics: A Multi-Model Analysis. Atmospheric Chemistry and Physics 13 (5):2793-2825.

Karantounias, Anastasios G. 2013. Managing Pessimistic Expectations and Fiscal Policy. Theoretical Economics 8 (1):193-231.

Keynes, John Maynard. 1937. The General Theory of Employment. Quarterly Journal of Economics $51(2): 209-223$. 
Klibanoff, Peter, Massimo Marinacci, and Sujoy Mukerji. 2005. A Smooth Model of Decision Making Under Uncertainty. Econometrica 73 (6):1849-1892.

Knight, Frank H. 1921. Risk, Uncertainty, and Profit. Houghton Mifflin.

Kreps, David M. and Evan L. Porteus. 1978. Temporal Resolution of Uncertainty and Dynamic Choice. Econometrica 46 (1):185-200.

Lemoine, Derek and Christian P. Traeger. 2016. Ambiguous tipping points. Journal of Economic Behavior and Organization 132:5-18.

Lettau, Martin and Jessica Wachter. 2007. Why is Long-Horizon Equity Less Risky? A DurationBased Explanation of the Value Premium. Journal of Finance 62:55-92.

Li, Xin, Borghan Narajabad, and Ted Temzelides. 2016. Robust dynamic energy use and climate change. Quantitative Economics 7 (3):821-857.

Lucas, Robert E. and Edward C. Prescott. 1971. Investment Under Uncertainty. Econometrica 39 (5):659-681.

Maccheroni, Fabio, Massimo Marinacci, and Aldo Rustichini. 2006. Dynamic Variational Preferences. Journal of Economic Theory 128 (1):4-44.

MacDougall, Andrew H., Neil C. Swart, and Reto Knutti. 2017. The Uncertainty in the Transient Climate Response to Cumulative CO2 Emissions Arising from the Uncertainty in Physical Climate Parameters. Journal of Climate 30 (2):813-827.

Matthews, H. Damon, Natahan P. Gillett, Peter A. Scott, and Kristen Zickfeld. 2009. The Proportionality of Global Warming to Cumulative Carbon Emissions. Nature 459:829-832.

Miao, Jianjun and Alejandro Rivera. 2016. Robust Contracts in Continuous Time. Econometrica 84 (4):1405-1440.

Millner, Antony, Simon Dietz, and Geoffrey Heal. 2013. Scientific Ambiguity and Climate Policy. Environmental and Resource Economics 55 (1):21-46.

Morgan, M. Granger, Parth Vaishnav, Hadi Dowlatabadi, and Ines L. Azevedo. 2017. Rethiking the Social Cost of Carbon Dioxide. Issues in Science and Technology 33 (4):43-50.

Nordhaus, William D. 2008. A Question of Balance Weighing the Options on Global Warming Policies. New Haven, Connecticut: Yale University Press.

. 2017. Revisiting the Social Cost of Carbon. Proceedings of the National Academy of Sciences 114 (7):1518-1523.

Nordhaus, William D. and Andrew Moffatt. 2017. A Survey of Global Impacts of Climate Change: Replication, Survey Methods, and a Statistical Analysis. Tech. rep., SSRN. 
Parker, Jonathan A. and Christian Julliard. 2003. Consumption Risk and Expected Stock Returns. American Economic Review Papers and Proceedings 93:376-382.

Petersen, Ian R., Matthew R. James, and Paul Dupuis. 2000. Minimax Optimal Control of Stochastic Uncertain Systems with Relative Entropy Constraints. IEEE Transactions on Automatic Control 45:398-412.

Pindyck, Robert S. 2013. Climate Change Policy: What Do the Models Tell Us? Journal of Economic Literature 51 (3):860-872.

Qin, Likuan and Vadim Linetsky. 2020. Long-Term Risk: A Martingale Approach. Econometrica 85 (1):299-312.

Ricke, Katharine L. and Ken Caldeira. 2014. Maximum Warming Occurs about One Decade After a Carbon Dioxide Emission. Environmental Research Letters 9 (12):1-8.

Ross, Stephen A. 2015. The Recovery Theorem. The Journal of Finance 70 (2):615-648.

Rubinstein, M. 1976. The Valuation of Uncertain Income Streams and the Pricing of Options. The Bell Journal of Economics 7:407-425.

Saltelli, Andrea and Mario Giampietro. 2017. What is Wrong with Evidence Based Policy, and How Can it be Improved? Futures 91 (August):62-71.

van Binsbergen, Jules, Michael Brandt, and Ralph Koijen. 2012. On the Timing and Pricing of Dividends. American Economic Review 102 (4):1596-1618.

Weitzman, Martin L. 2012. GHG Targets as Insurance Against Catastrophic Climate Damages. Journal of Public Economic Theory 14 (2):221-244. 